\title{
SOLUTION OF BETHE-SALPETER EQUATION WITH PADE APPROXIMANTS
}

\author{
H. M. NIELAND \\ Institute for Theoretical Physics, University of Nijmegen, The Netherlands \\ and \\ J. A. TJON \\ Institute for Theoretical Physics, University of Utrecht, The Netherlands
}

Received 19 June 1968

\begin{abstract}
The Bethe-Salpeter equation for the $\pi \mathrm{N}$ system in the ladder approximation with $\mathrm{N}$ exchange is solved in the elastic region using Pade approximants. It is shown that the lowest order diagonal approximant [1,1] is a bad approximation for this case.
\end{abstract}

Recently there has been interest in the application of Padé approximants within the domain of particle physics $[1,2]$. In this method the scattering amplitude $T(g)$ for a given value of the coupling constant $g$ is approximated by a quotient of two polynomials $P_{M}(g)$ and $Q_{N}(g)$ of order $M$ and $N$ respectively; their coefficients are determined from the power series expansion of $T(g)$ at $g=0$ [3]. Results obtained in non-relativistic potential scattering for certain potentials showed that the sequence of diagonal approximants $(M=N)$, usually denoted by $[N, N]$, converges rapidly with increasing $N$ [4]. Moreover, the first approximant $[1,1]$ turned out to be already a reasonable approximation to the exact solution, even for rather large coupling constants. In view of the possible applications of this method to the study of the dynamics of strongly interacting particles it is of some interest to study in a systematic way its usefulness in an actual field theoretical model.

In this note we present some results on the solutions of the Bethe-Salpeter equation for $\pi \mathrm{N}$ scattering in the ladder approximation with $\mathrm{N}$

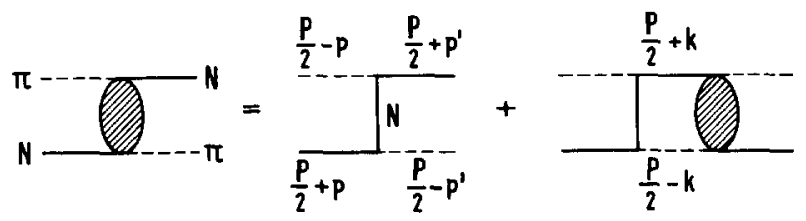

Fig. 1. The Bethe-Salpeter equation in diagrammatic form. $P$ is the total $4-$ momentum; in the c.m. system $P=(0,0,0, \sqrt{s})$.

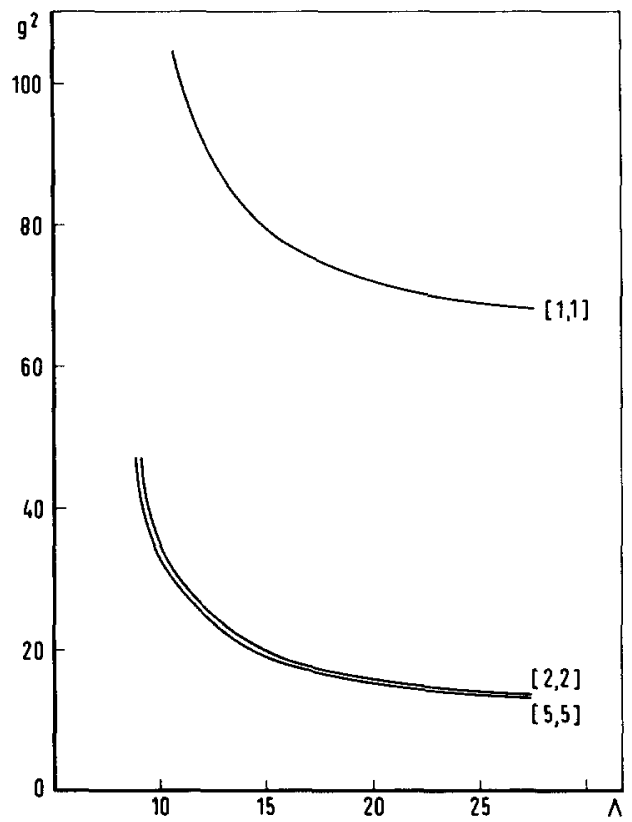

Fig. 2. $g^{2}$ versus $\Lambda$ at elastic threshold $s=61.6$ for some approximants. On this scale $[3,3]$ and $[4,4]$ coincide with $[5,5]$.

exchange and pseudoscalar interaction. The equation in diagrammatic form is shown in fig. 1. The corresponding integral equation for the scattering amplitude $T$ in momentum space is 


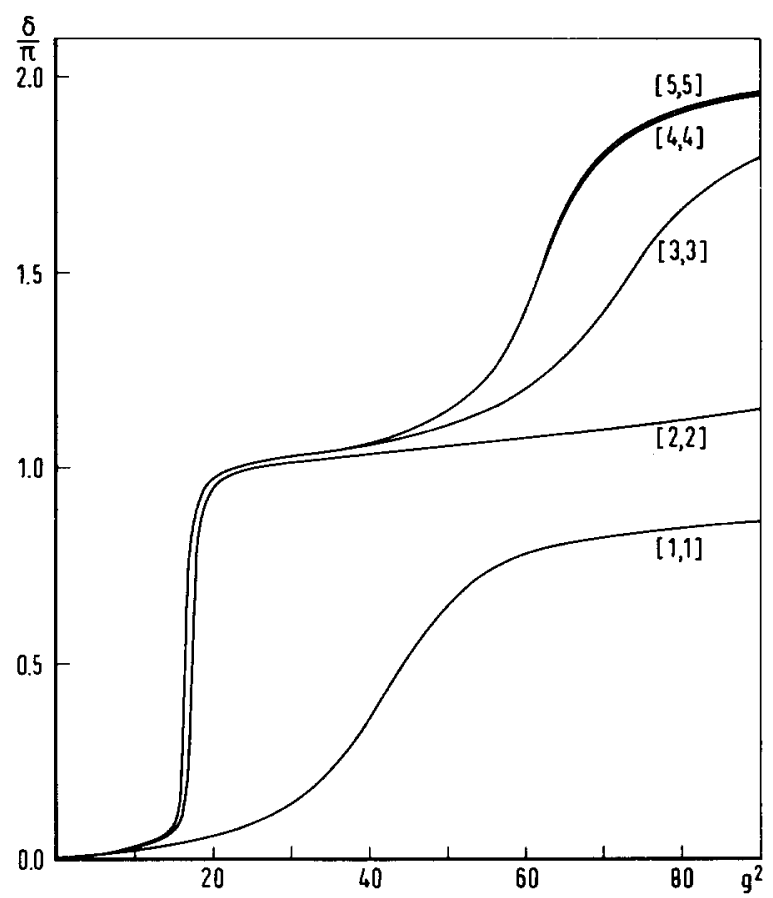

Fig. 3. The phase shift versus $g^{2}$ at $s=70$ and $\Lambda=15$.

given by

$$
\begin{array}{r}
T\left(p, p^{\prime}\right)=-4 \pi g^{2}\left[\mathrm{i} \gamma\left(p+p^{\prime}\right)+M\right] \Delta\left(p+p^{\prime}\right)+ \\
-\mathrm{i} 4 \pi g^{2} \int \frac{\mathrm{d} 4 k}{(2 \pi)^{4}}[\mathrm{i} \gamma(p+k)+M] \Delta(p+k) \times \\
\quad \times\left[\mathrm{i} \gamma\left(\frac{1}{2} P+k\right)-M\right] G(k) T\left(k, p^{\prime}\right)
\end{array}
$$

with

$$
\begin{aligned}
& \Delta(k)=\left[k^{2}+M^{2}-\mathrm{i} \epsilon\right]^{-1} \\
& G(k)=\left[\left(\frac{1}{2} P+k\right)^{2}+M^{2}-\mathrm{i} \epsilon\right]^{-1}\left[\left(\frac{1}{2} P-k\right)^{2}+1-\mathrm{i} \epsilon\right]^{-1} .
\end{aligned}
$$

We have set here $m_{\pi}=1$. Furthermore the coupling constant is defined in such a way that the experimental value of $g^{2}$ is given by 14.5 .

Each term in the perturbation series solution of eq. (1) is finite as can easily be inferred from power counting arguments. However, the integral equation itself is marginally singular. For this reason a relativistic cutoff is introduced in the interaction by making in eq. (1) the substitution $\Delta(k) \rightarrow \Delta_{\Lambda}(k)=\left[k^{2}+M^{2}-\mathrm{i} \epsilon\right]^{-1}-\left[k^{2}+\Lambda^{2}-\mathrm{i} \epsilon\right]^{-1}$. Introduction of a complete set of helicity spinors in eq. (1) leads after partial wave decomposition to a pair of coupled integral equations in spinor space which are of the form

$$
\begin{aligned}
T_{m}\left(p, p_{\mathrm{o}}\right)=V_{m}\left(p, p_{\mathrm{O}}\right) & +\sum_{n=1}^{2} \int_{\mathrm{O}}^{\infty} \mathrm{d} k \int_{-\infty}^{+\infty} \mathrm{d} k_{\mathrm{O}} \times \\
& \times K_{m n}\left(p, p_{\mathrm{O}} \mid k, k_{\mathrm{O}}\right) T_{n}\left(k, k_{\mathrm{O}}\right) .
\end{aligned}
$$

In eq. (2) only the integration and spinor variables are written out explicitly.

In order to solve these equations numerically a Wick rotation has been performed in the $k_{0}$ variable from the real to the imaginary axis, a procedure already used in the case of scalar particles [5]. The remaining singularities can be handled numerically. No extra complications arise as long as $\Lambda>M$. The resulting equations have been iterated on a IBM 360-50 using an integration with 8 Gaussian points in each variable. The accuracy of the coefficients obtained in this way has been tested to be better than $1 \%$ by increasing the number of integration points. An independent check was also made on the box diagram contribution by calculating it in a different way. The solution of Bethe-Salpeter equation was obtained by constructing diagonal Padé approximants from the coefficients of this perturbation series expansion in $g^{2}$. One of the main advantages of these diagonal approximants is that elastic unitarity is automatically satisfied.

Some results which are representative for the applicability of this method are given in figs. 2, 3 and 4 . Specifically the $\mathrm{p}_{33}$ partial wave is considered here. In fig. 2 the coupling constant for which the first bound state occurs at elastic thres-

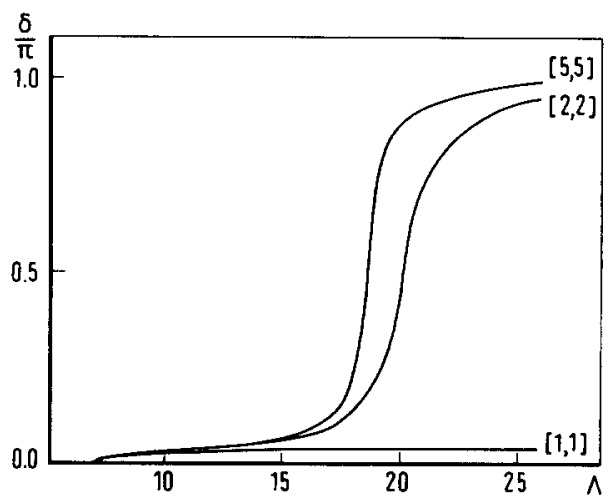

Fig. 4. The phase shift versus $\Lambda$ at $s=70$ and $g^{2}=$ $=14.5$. On this scale $[3,3]$ and $[4,4]$ coincide with $[5,5]$. 
hold is given as a function of the cutoff parameter $\Lambda$. It shows a rapid convergence of the sequence of $[N, N]$ approximants. The first approximant $[1,1]$ however gives results which differ considerably from the exact values. In contrast to the fact that the $[1,1]$ approximant cannot produce, even for infinite cutoff, a bound state below $g \mathbf{2} \approx 66$, the exact solution already gives a bound state for $\Lambda>22$ at $g^{2}=14.5$. As a consequence the $\mathrm{N}_{33}^{*}$ can even be realised here in the Bethe-Salpeter equation as a bound state. Of course it can be obtained as a resonance by adapting the cutoff parameter.

For a given $\Lambda$ the sequence of $[N, N]$ approximants converges slower for increasing coupling constant. This is shown fig. 3 where the phase shift is plotted as a function of $g^{2}$ at $s=70$ and $\Lambda=15$. For this value of the cutoff a second bound state appears at $g^{2}=80$. Even in this region of $g^{2}$ the $[4,4]$ and $[5,5]$ approximants are still very close to each other. Finally in fig. 4 the phase shift is given as a function of $\Lambda$ at $s=$ $=70$ and $g^{2}=14.5$. Here too it is found that the $[1,1]$ approximant is a very poor approximation.

To summarize, we may conclude that although the Padé method can be used to solve the BetheSalpeter equation for the $\pi \mathrm{N}$ system for intermediate strong interaction higher order approxi- mants than $[1,1]$ are needed. It is in contrast to the case of scalar particles where the $[1,1]$ approximant already gives a reasonable description [6]. This difference may be ascribed to the singular nature of the one nucleon exchange force in the $\pi \mathrm{N}$ case.

\section{References}

1. L. A. Copley and D. Masson, Phys. Rev. 164 (1967) 2059;

D. Bessis and M. Pusterla, Nuovo Cimento 54 (1968) 243.

2. E. Remiddi, M. Pusterla and J.A. Mignaco, Phys. Letters 27 (1968) 34;

J.L.Gammel, M.T. Menzel and J.J.Kubis, Los Alamos Scientific Laboratory preprint.

3. For a review of the theory of Padé approximants, see G. H. Baker jr. in Advances in Theor. Physics vol. 1, 1965.

4. J.L.Gammel and F.A.McDonald, Phys. Rev. 142 (1966) 1145.

5. M.J.Levine, J.Wright and J.A.Tjon, Phys. Rev. 154 (1967) 1433.

6. J. Nutall, Phys. Letters 23 (1966) 492. 